\title{
Development and application of hydrogen energy and fuel cell vehicle
}

\author{
Ma Shuo
}

Wuhan Jiaotong Vocational College Automotive Engineering College Hubei province Wuhan City430065

\begin{abstract}
: is currently,continued growth in global energy demand and dwindling fossil fuel resource reserves,and environmental security issues are gradually highlighted,makes it possible to create aClean and sustainable energy systems have become an urgent need for national security strategies for the future ofnations.awide range of hydrogen sources,use clean and can thenhealth,is ideal for traditional fossil fuels alternative energy.This article mainly introduces the development of hydrogen energy and hydrogen production and hydrogen storage technology,and analyzing hydrogen energyTechnologies in the development of automobiles,-fuel cell car.To resolve the energy problems that are currently facing.
\end{abstract}

Keywords: hydrogen Energy;hydrogen production;hydrogen Storage;fuel cell car

\section{Overview}

\section{1 article Overview}

In terms of theevolution of human fuels,hydrogen will be humanclass future fuel.TheHuman fuel evolution has undergone a process fromthe the Thecoal to oil to the natural gas again,So with the hydrogen gasContinuous application,Future fuel must be hydrogen energy. ${ }^{[1]} /$ humanevery fuel evolution in history has been accompanied by human civilization.Step and development,then, The future of the hydrogen energy era certainlychanges our human lifestyle.hydrogen is the cleanestfuel,Unit mass of hydrogen with three times times the gasoline'scalorific value. ${ }^{[2]}$ but,hydrogen is not a natural resource,but peopleMakeResources,cost of production and extraction of hydrogen, thecost of making hydrogen will also be nearly three times times that ofpetrol,thereforenowhere excessive costs increase during storage and use of hydrogenTheapplication of hydrogen is unfavourable.There are many problemswith the current hydrogen energyLive application,the supply and demand relationship of the market is notis the main bottleneck inhibiting hydrogen development,Real limit hydrogen energyThe reason why is widely used is also how to efficiently process hydrogen.and efficient low-power storage of hydrogen and safe transport and managementhydrogen etc.,additional facilities for hydrogen resources,thansuch as hydrogen station construction,all constitute limits of hydrogen usefactor.

\section{2 discovery of hydrogen gas}

Theearliest documented reference to hydrogen is inthecentury,Swiss Alchemist Palachersus using acid with certain goldreaction prepared hydrogen gas,But because people at that time haveA limited understandingof the hydrogen "'" gas,todirectly divide this new gasintoair.The first clear definition of hydrogen is a distinguished chemist.lavoisier,Name the combustible gashydrogen ${ }^{[5]}$

hydrogen is the richest substance in the universe,hydrogen on earthexists mainly in the form of acombination, such as water,carbon hydridecomplexes and various organic compounds.hydrogen inK (-2535The cryogenic conditions of theC)can be liquefied.due to the thermal conductivity of hydrogen,spread faster,with oxygen burning,Its propagation speed is aalkanes4times, hydrogen and oxygen burning highly susceptible to explosive reactions. The main technical

\section{Copyright (C).}

This is an open-access article distributed under the terms of the Creative Commons Attribution Unported License

(http://creativecommons.org/licenses/by-nc/4.0/), which permits unrestricted use, distribution, and reproduction in any medium, provided the original work is properly cited. 
problem with hydrogen combustion is how to control the ignitionBurn speed.hydrogen is a more common reductant,so,hydrogen is widely used in the chemical field.

\subsection{Research on hydrogen energy}

Because hydrogen has a wide range of sources,Unit mass EnergyHigh density,use pollution-free and reproducible features,hydrogenhas been regarded as the best alternative to traditional fossil fuels the future of the""Energy.anin-depth study of to address the energy crisisand environmental sustainability issues have far-reaching strategic implicationssemantic.All countries in the world have done a lot of research on hydrogen.work,such as U.S.,Japan,German,UK,RussianRoss,South Korea and other countries set up institutions to study hydrogen gas,,theCost of the hydrogen research project is increasing.,China also takes hydrogen as part of future strategic energy,domestic Tsinghua University,Dalian Chemical Institute,Zhejiang University,sameUniversity of Jinan,Universities in hydrogen researchhas a wealth of experience.current,Research on hydrogen mainThe is set in the following areas:

(1)hydrogen production, Research in this area mainly involvesand how to reduce the cost of hydrogen production and improvethe manufacturing processlevel etc;

(2)Storage of hydrogen, Themain research in this area isHow to increase the energy density of hydrogen storage and develop newhydrogenstorage materials and lower storage costs;

(3)use of hydrogen, Research in this area is mainly concerned withand how to increase the efficiency of hydrogen use;

also,andSafety management and transport of hydrogen.,HydrogenationStation construction and related laws and regulations research.this""is not a specific system for the hydrogen industry doing research,instead of thehydrogen production,Store and apply to the carGeneral description,So readers can read this article,ToA preliminary understanding of hydrogen-related knowledge.

\section{2. hydrogen production}

\section{1 electrolysis of water for hydrogen production}

electrolysis of water hydrogen production process is very ancient hydrogen production method,Craft Simple,production process automation High,Easy,High purity of hydrogen made by,to reach99\% 99.9\%.current,Domestic Electrolysis water hydrogen technologyTheoperation is relatively complete.theoretically,as long as voltage reaches1.229Vtodo electrolytic water,but because hydrogen and oxygen have been generatedthe voltage in the process exists,,electrolyte interference and other resistorsreason,cause actual electrolytic voltage to be higher than1.229V.High power consumption in electrolytic water,actual system $1 \mathrm{~m}^{3}$ (subscripthydrogen consumes less power than $5 \mathrm{kw}^{\prime} \mathrm{h}^{\prime} \mathrm{m}^{-3}$,

Fortoday, The cost of electrolytic water for hydrogen production is still a comparisonhigh.Despite serious energy dissipation problems with electrolytic water hydrogen,But for our country,still has a broad development beforeKing,Our hydropower,Rich in hydroelectric resources,in the south-westThe area has a powerful advantage, How to use our geographical areafield differences and how to take advantage of the power station's low timeResidual electricity has always been a problem for scholars to study.,using electrolysisThemethod of making hydrogen by water can solve these problems,currently Icountries are also actively promoting"Small hydro Fuel eco-building"Set Project""construction.

\section{2 hydrogen production from fossilfuels(reforming gas hydrogen production)}

fossil feedstock for hydrogen production is coal,Coke,Oil,Natural gas and methanol derived from them,ammonia etc asraw material for hydrogen production,currently around the world aboutthehydrogen for the A. is made from this method.for hydrocarbon fossilsrawmaterials hydrogen production mainly includes steam reforming,Self-thermal rebuild(oxidative reorganization),hydrocarbon decomposition,light hydrocarbon selective oxidationhydrogen and plasma steam reforming five different manufacturing processes.Current domestic and foreign large-scale hydrogen production plant with hydrocarbon steam transferto the main.This method is either in the Catalyst 
preparation,processTechnology and reliability of equipment are already very mature,while other technologies are still in the research phase.ammonia decomposition hydrogen ismanyfloat glass Enterprises Adopt main hydrogen production method,BaseThis process is liquid ammonia by preheating,evaporating to ammonia,at high temperaturecatalyze decomposition into ammonia and nitrogen,then adsorbed purification,can get high purity hydrogen in separation,but ammonia decomposition systemhydrogen also has some deficiencies,For example, high reaction temperature,requireslots of calories,consuming energy,as well as for reactors and switchingHot material requirements are higher;Second is liquid ammonia transport beltThe cost increases for and security issues.

\subsection{Bio-hydrogen production}

the Bio-hydrogen technology is the use of microorganisms under certain conditionsto produce hydrogen for hydrogen production.dueto the traditional hydrogen production method exists the problem of consuming non-renewable energy.,no longer meet future social development requirements,Bio-hydrogen production technologyis getting more attention from countries, where Germany,Japan,US,Israel,Russia,Britain has invested a lot of manpower,Resources for this study,United States annual study BiologyThe single cost of hydrogen production is as high as millions ofUSD,and Japanput in more.These countries have established specialized institutions,Establish the development plan of biological hydrogen production,for bio-Hydrogen Basicsa great deal of research has been done and applied technology.Current research workmainly focus on two aspects:one is,seek Highyield-producing microorganisms; Two: Research on hydrogen production process.

\subsection{Solar hydrogen production}

Human use of solar energy can be traced back tothe3000years ago,and turning solar energy into a power and powertime onlyyear,with the advent of science and technology nowstepand energy crisis increasingly grim,Human Solar energyThe development and utilization of the has also been accelerated.,which uses solar energy systemhydrogen is the way humans use solar energy.currently using toohydrogen productionby solar thermal decomposition water hydrogen,Solar PV generation hydrogen,photocatalytic photocatalytic photolysishydrogen,,Solar bio-hydrogen, etc..

\section{3. hydrogen application in automobiles}

\section{1 hydrogen burning technology}

hydrogen may be an ideal energy,because it canget from non fossil raw materials,combustion of hydrogen does not produceCO-,HC,SOx,Carbon smoke pollutants,in aclean hydrogen enginewitha well-designed,does not cause sulfuric acid deposition,productioncarcinogenic substances such as benzene.So the research on hydrogen-burning technologyhas been cited as ageneral interest of many national researchers inthe.The main way for thecurrent hydrogentobe the direct fuel for a car's internal combustion engine is theTwo kinds, One is a hydrogen gas that uses hydrogen directly as fuelmotives, Two is in traditional internal combustion engine fuel(as gasoline,Diesel)Adding trace amounts of hydrogen to the.

compared to other traditional fossil fuels, hydrogen as the startingA notable feature of fuel for machines:

(1)compared to other fuels,at a wider temperature andpressure range,hydrogen has a higher flame in the tankMulticast Speed;

(2)The mixture of the ignited hydrogen engine running is strongLowerlimit than other fuel engines;

(3)Dilute mixed gas concentration, hydrogen combustion engine onStop places,Fast burning energy release rate make hydrogen-Airmixture burns quickly,causes higher efficiency output;

(4)hydrogen fuel engines have lower emission pollution problems;

(5)Hydrogen's fast burning rate makes it suitable for high speedengine;

(6)Change the hydrogen fuel engine ignition time is improvedengine performance and effective way to avoid detonation;

otherwise,hydrogen fuel engines and workingoverthread Loop lower,High octane,Cold start performance,,heat loss 
small,Efficiency high features.

\section{2 fuel Cell}

A fuel cell car is the main that hydrogen can apply to automobilesto form.fuel cell through hydrogen with air or oxygen-generateElectric Energy drive motor Drive auto Linesail,fuel cell is equivalent to an energy conversion device, ,Convert chemical energy to electrical energy.iscomparedto thetraditional internal combustion engine andthe batteryThefuel cell has many advantages.dueto fuel convert chemical energy directly to electrical energy,Isfar more efficientthanhigher than internal combustion engine;fuel cell is ideal all solid-state machinerymechanism system,Such systems are potentially highly reliableand long life,andrunning very quiet, andalso do not produce such asNOx,,Sox, and particulate emissions;Compare traditional battery,burnMaterial battery allows arbitrary scaling between power and capacity,itsThe dimension can also be from1tile level(phone)do MW(moveForce factory.

\section{Epilogue}

Inrecentyears,Development and utilization of hydrogen energy at home and abroaddo a lot of theoretical and experimental research,and get very explicitachievements.Although this progress is encouraging,but away from hydrogenExtensive commercial use of there's a long distance., andMany issues need to be resolved,For example how to find from renewableresources to make hydrogen and reduce the cost of hydrogen production; How to increase the storage density of hydrogen;How to improve hydrogen'sManaging and applying security issues; How to optimize the for hydrogen carsdesign and solve the current technology used by hydrogen in carsbarriers etc.only on these issues the overall,Reconcile,Deep Research,To keep the hydrogen safe,,secure,apply,To fundamentally address our future descendants.Future generation's energy security issues.4; wear

\section{References}

1. MaoZongqiang.hydrogen Energy-21Green Energy for the century[M].NorthBeijing:Chemical Industry publishing house, 2005:10-21.

2. GaoMinze.Research on China's new energy automobile industry[D].GilletteForest University,2013.

3. E.MacA.Gray.hydrogenStorage-statusandprospects.advancesinappliedceramics2007;106,25-28.

4. DanZhao,daqiangYuan,hong-cai Zhou.the currentstatustohydrogenstorageinmetal-organicframeworks.Energy\&environmental2008;1,222-235.

5. Changan,White Pine,Shangjian.Status of hydrogen storage methodsand development[J]].Ship-proof,2009,1:52-56. 